\title{
RELACIONES INTERNACIONALES
}
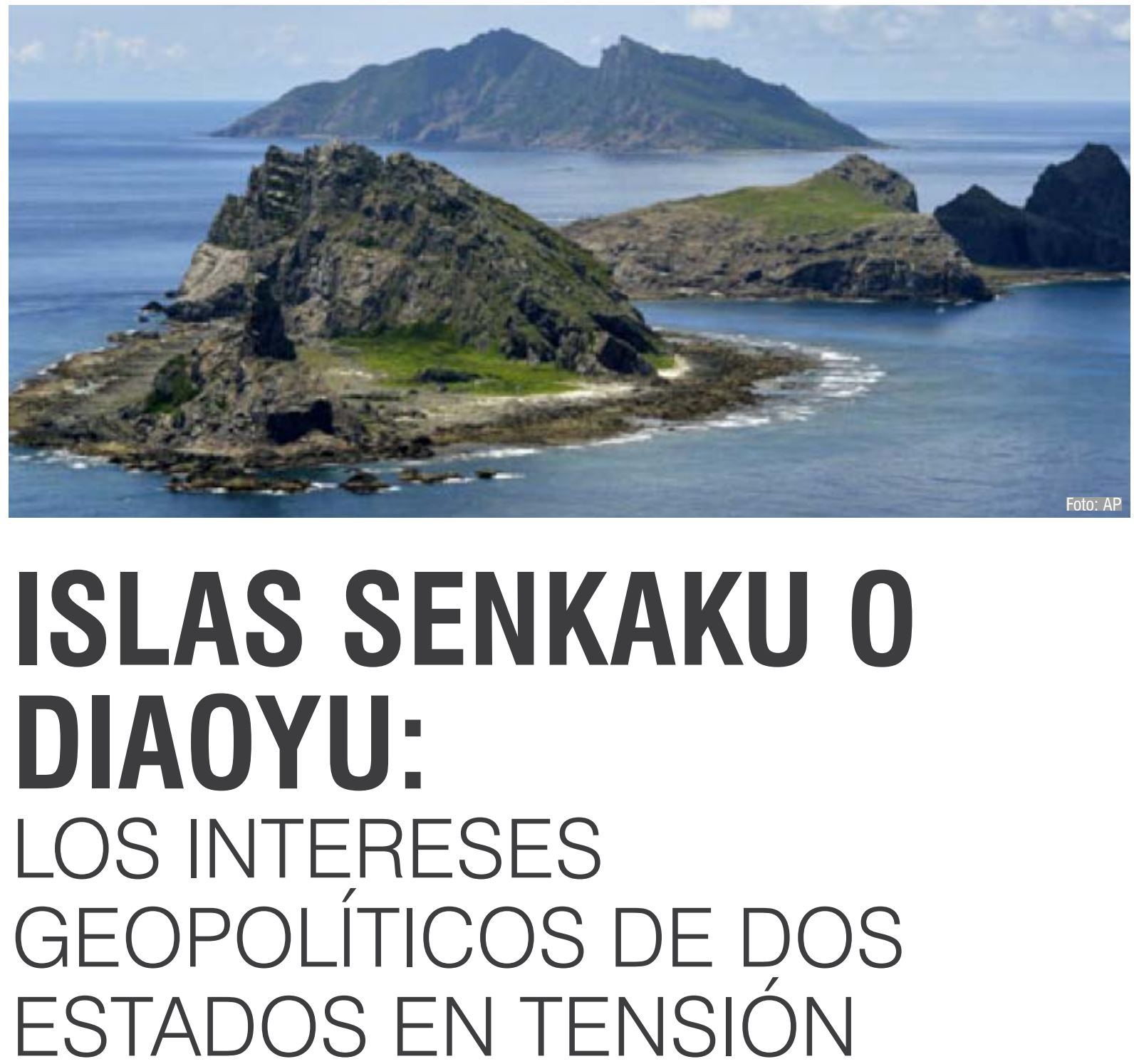

$>>$

Daniel Elifonso Cardona Ruiz

Estudiante de Negocios

Internacionales, Universidad EAFIT.

E-mail: dcard017@eafit.edu.co

\section{Resumen}

La disputa fronteriza entre Japón y China sobre las islas Diaoyu o Senkaku ha estado presente por más de cuarenta años. Este conflicto está caracterizado por ser cíclico, pues por varios años se puede dejar de hablar del asunto, y de un momento a otro, acciones por parte de uno de los dos países generan inmediato descontento en el otro, que va desde simples protestas callejeras a xenofobia y amenazas de limitación comercial. Con el fin de entender mejor la situación, el presente artículo presenta los hechos históricos más importantes a lo largo de la disputa, al igual que analiza las acciones tomadas por ambas partes en los últimos meses.

\section{Palabras clave}

China, Japón, Islas Senkaku, Islas Diaoyu, geopolítica, Estados Unidos, disputa territorial. 
Es medio día del 15 de Septiembre en la cuidad portera de Qingdao, en la provincia china de Shandong. Decenas de personas corren disipadas por los gases lacrimógenos que lanza la policía para tratar de disipar a la horda de enfurecidos protestantes que acaban de incendiar una planta de producción de la compañía Panasonic. Protestas esporádicas se han convertido en disturbios violentos formados por cientos de personas en más de una docena de ciudades chinas. Fábricas han sido incendiadas, supermercados y tiendas han sido saqueadas, embajadas y consulados han presenciado enormes protestas a sus afueras. El común denominador de todo este caos: sentimientos anti-japoneses en China.

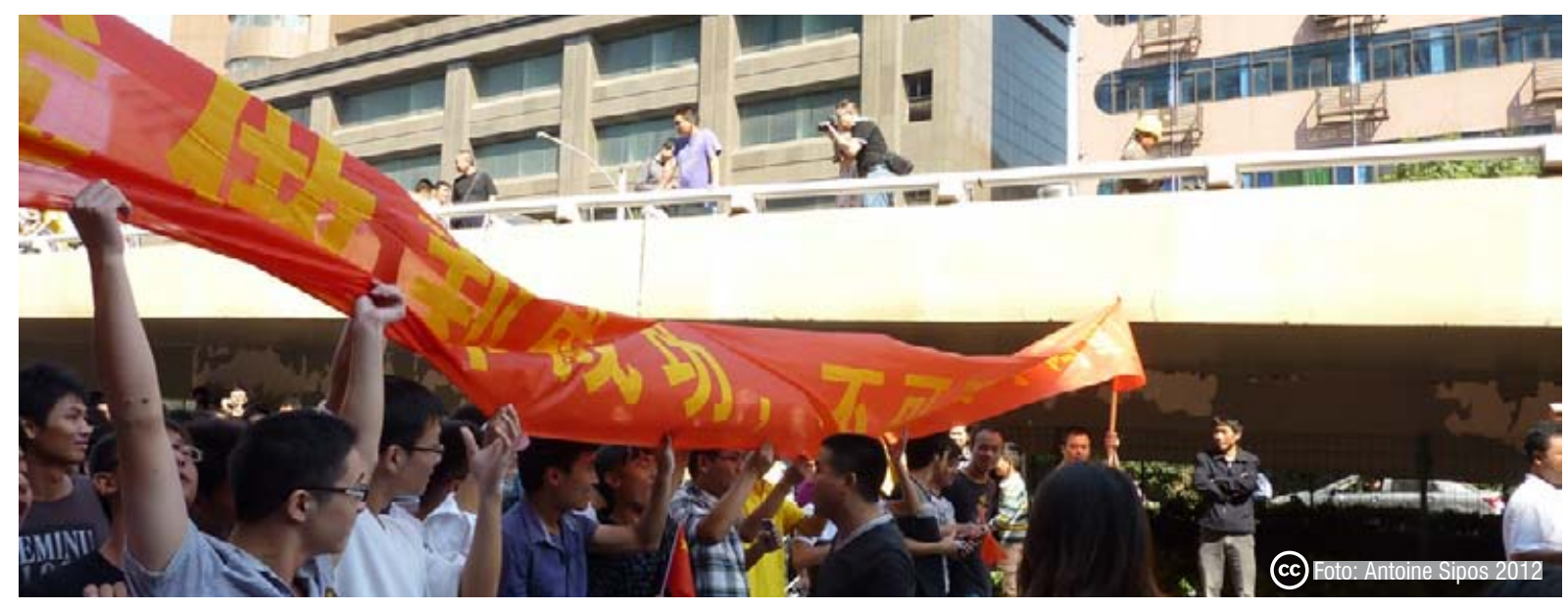

Antecedentes: Islas pequeñas. Fuentes de grandes problemas

Después de la victoria japonesa sobre China en la primera guerra sinojaponesa (1894-1895), el país nipón se apoderó de la isla de Taiwán y de sus territorios anexos, incluyendo las islas Diaoyu o Senkaku, como son conocidas en China y Japón respectivamente. En 1896, el gobierno del Japón cede las islas Senkaku al empresario Tatsushiro Koga quien desarrolla una empresa de pesca de bonito en la zona. Sin embargo, la empresa quiebra en 1940 y las islas vuelven a estar deshabitadas, siendo aun propiedad de la familia del señor Koga quien muere en 1918 (Maya, 2010).

Luego de la rendición de Japón en 1945, los territorios antes conquistados por el Imperio del sol naciente pasan a control de los países aliados, siendo Estados Unidos el encargado de la administración de dichas islas. Por medio del tratado de San Francisco de 1951 (United Nations, 1951), Taiwán es devuelto a China, pero las islas Senkaku siguen siendo administradas por Estados Unidos hasta 1972 cuando son devueltos al gobierno de Japón (Tratado de Okinawa, 1971).

Uno se preguntaría porque tanta conmoción sobre unas islas que han estado deshabitadas por años y que territorialmente no representan un gran área. La respuesta es que en 1971 se descubrió que en el subsuelo de estas islas podría llegar a haber hasta 100 mil millones de barriles de petróleo de acuerdo con la Administración de Información Energética (EIA) de Estados Unidos (Ogura\& Mullen, 2012). Esto llevo inmediatamente a que China y Taiwán demostraran sus intereses de retomar el dominio de las islas.

Desde entonces numerosos roces entre Japón y China han surgido. Los más comunes son a causa de incursiones de barcos pesqueros en aguas cercanas a las islas y el desembarco de activistas chinos o taiwaneses como forma de protesta y que comúnmente terminan con su arresto por parte de la marina japonesa. 
Las protestas a lo largo de toda China, en contra de Japón, volvieron a reanudarse cuando el pasado mes de septiembre el gobierno nipón decidió comprarle tres de las islas a un industrial japonés por un valor de más de 2500 millones de yenes (26 millones de dólares) (Gayathri, 2012) con el objetivo de nacionalizar las islas. Inmediatamente se generaron manifestaciones violentas anti japonesas en China y Taiwán, elevando las tensiones entre las partes.

Estas disputas sobre los recursos que se encuentran en las islas Senkaku para los japoneses o Diaoyu para los chinos, podría afectar más que las relaciones entre ambos países; el gran perdedor seria la economía mundial. Japón es la tercera economía del mundo, mientras que China, desde el 2010, es la segunda. Japón tiene una gran dependencia económica de China, especialmente en materia de exportación, manufactura, y materias primas. Las tierras raras son de especial importancia entre estas últimas. Las tierras raras son un grupo de 17 elementos químicos con nombres imposibles de pronunciar, pero fundamentales para la producción de motores, aires acondicionados y computadoras (Japanese Firms, 2012).China genera el 95\% de la oferta mundial de tierras raras, y desde el 2010 cuando un barco chino colisionó con un barco guardacostas de Japón en inmediaciones de las islas Senkaku, la exportación de estos materiales hacia Japón se ha visto drásticamente reducida, afectando la producción de empresas niponas como Toyota, Mitsubishi y Panasonic, quienes tratan de buscar alternativas a la dependencia de las tierras raras importadas desde China. Unas disminución en la producción de las fábricas japonesas en China significaría no sólo una reducción en el intercambio comercial entre ambas naciones, sino que podría afectar el abastecimiento de productos japoneses en otras partes del mundo.

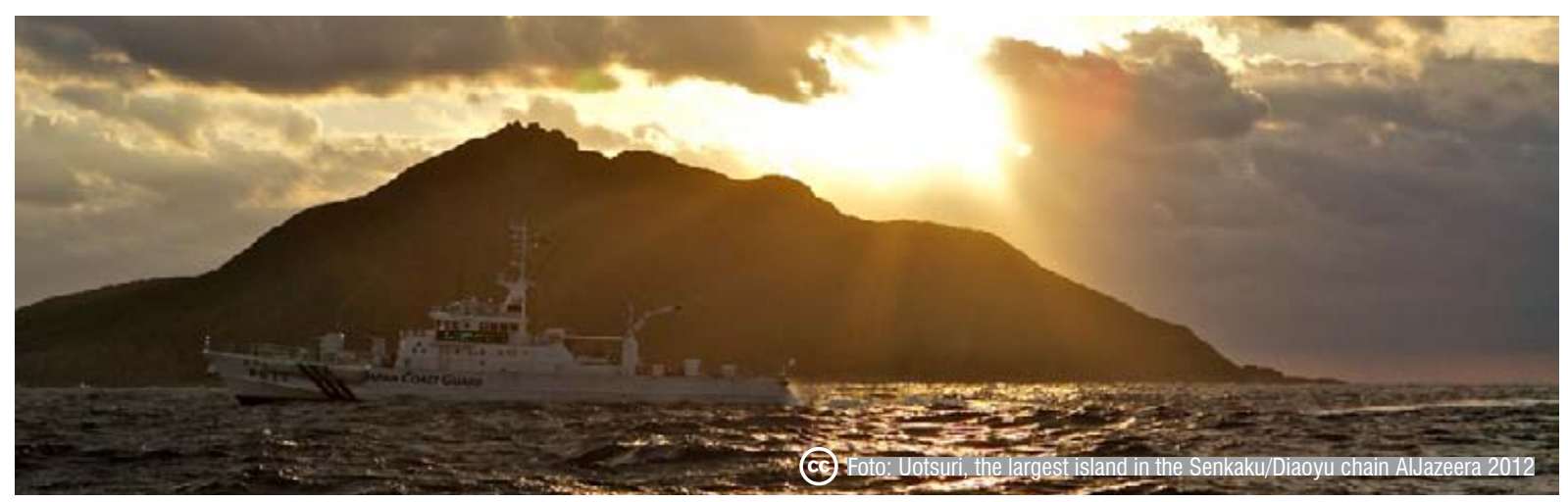

\section{"Boicoteen los productos japoneses" dicen los medios}

Recién ocurridas las primeras demostraciones anti japonesas por los conflictos territoriales entre China y Japón en 2012, los medios estatales chinos promovieron boicots contra bienes y establecimientos japoneses. Periódicos chinos pusieron en sus páginas listas con nombres de empresas japonesas para que sus lectores los dejaran de comprar. A su vez, varios canales de televisión estatales dejaron de mostrar avisos publicitarios de marcas como Sony, Panasonic, entre otras.

Estos mensajes fueron sólo parte de la euforia del momento, ya que la dependencia económica entre ambos gigantes del comercio mundial pudo más que los sentimientos nacionalistas. En una semana de iniciadas las revueltas, los medios estatales estaban llamando a la calma y a no utilizar sentimientos patrióticos como justificación a la perturbación del orden público. Esta retractación también llegó a los medios de comunicación digitales y redes sociales como Sina Weibo (sitio web chino parecido a Twitter) en donde las búsquedas de temas relacionados con las protestas anti-japonesas habían sido bloqueadas. 
Pero los usos de medios de comunicación para favorecer un determinado punto de vista sobre la situación de las islas no es de uso exclusivo de China. En el último mes, Japón ha emprendido una campaña diplomática para reforzar sus reclamos sobre las Islas Senkaku. Varios documentos oficiales explicando la situación han sido creados por el gobierno japonés, entre ellos el llamado "Tres verdades sobre las islas Senkaku" y repartidos a todas las sedes diplomáticas del gobierno nipón en el mundo. En ellos, se hace referencia a las islas como parte de territorio japonés a la vista del derecho internacional y de la historia. Esta campaña de guerra mediática cada vez se torna mas seria, con el Ministro de Relaciones japonés pidiendo unos 600 millones de yenes al gobierno, algo así como unos 7.5 millones de dólares, para dedicarlos a campañas de relaciones públicas con el objetivo de "mantener el territorio japonés" (Kyodo, 2012).

Los esfuerzos japoneses no sólo se limitan a medios de comunicación y documentos oficiales. Hasta la Asamblea General de las Naciones Unidas han llegado intentos de buscar apoyo internacional sobre el hecho, pero pocos son los resultados que se esperan de estas intervenciones, y en especial durante los debates generales, puesto que China, como miembro permanente del Consejo de Seguridad de la ONU, tiene una posición mas influyente dentro de la organización.

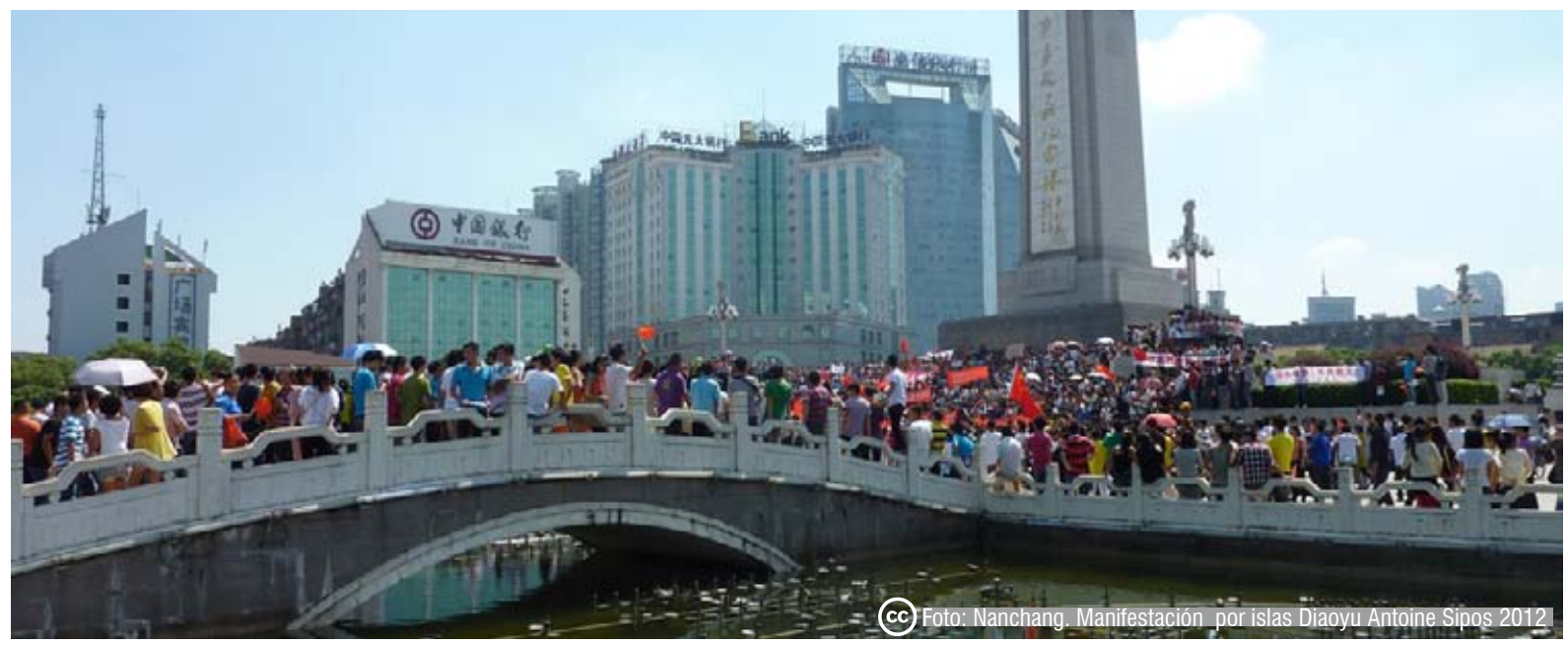

\section{"La respuesta es diplomática": otro movimiento geopolítico americano.}

Los intereses geopolíticos de Estados Unidos en Asia se encuentran entre las prioridades de la política exterior americana. Por un lado, uno de sus mayores socios comerciales y la segunda economía del mundo: China, y por el otro se encuentra una de sus piezas fundamentales en seguridad y principal aliado en la región: Japón.

Estados Unidos ha advertido a China que las islas Senkaku se encuentran dentro del Tratado de Seguridad entre EEUU y Japón, aunque mantiene su posición de no tomar partido en esta disputa territorial. Esto demuestra el delicado balance que Estados Unidos tiene que mantener entre su alianza con Japón y sus intentos de mejorar las relaciones con China. Estas declaraciones de neutralidad también tienen el objetivo de evitar que más atención de la necesaria sea puesta sobre el Tratado de Seguridad EEUU- Japón y generar la idea de una posible intervención militar del país norteamericano (Panetta tells China, 2012). 


\section{¿Y ahora qué?}

A pesar de los fuertes disturbios que ocurrieron en pasados meses y los sentimientos nacionalistas chinos exacerbados durante las protestas anti japonesas, todo parece volver a la normalidad, mientras que se repiten los mismo ciclos de conflictos pasados sobre las islas. Todo inicia con algún comportamiento japonés relacionado con las islas que genera ira en los chinos o taiwaneses, quienes inmediatamente, con alientos de los medios estatales, inician boicots nacionales contra empresas y productos japoneses. Luego cuando la idea de un escalamiento del conflicto aparece en ambos bandos, los dos gobiernos calman los ánimos e inician conversaciones bilaterales, como ocurre actualmente. El pasado 20 de octubre, el vice ministro japonés de asuntos exteriores Chikao Kawai visitó Shanghái para reunirse con su contraparte chino Zhan Zhijun para discutir asuntos relacionados con la isla.

Es muy improbable, dicen analistas, que el conflicto llegue a escalar. Es tal la interdependencia de ambos países orientales, que un conflicto sobre unas islas deshabitadas sería mas perjudicial que beneficioso para sus intereses económicos y políticos (AFP, 2012). Durante el 2011, Japón fue el segundo socio comercial de China, después de Estados Unidos, y las empresas japonesas emplean directa o indirectamente más de 10 millones de empleados chinos (Fackler\& Johnson, 2012). Lastimar esos lazos comerciales significaría una gran perdida económica para ambos países, no sólo en términos de intercambios comerciales, sino también en materia de empleos.

\section{Referencias Bibliográficas}

- $\quad$ AFP. (2012, Octubre 24). Japan, China officials met over islands dispute: Tokyo. Livemint. Consultado de http://www.livemint.com/Politics/T6pmuzkUoafDJZQF9MoJTN/Japan-China-officials-met-over-islanddispute-Tokyo.html

- Gayathri, A. (2012, Octubre 25). Chinese Ships Enter Disputed Waters Near Senkaku Again. International Business Times. Consultado de http://www.ibtimes.com/chinese-ships-enter-disputed-waters-nearsenkaku-again-853618

- Japanese Firms Join Forces to Reduce Reliance on China Exports (2012, Octubre 12). Want China Times. Consultado de http://www.wantchinatimes.com/news-subclass-cnt.aspx?id=20121012000061\&cid=1 102\&MainCatID = 11

- Kyodo. (2012, Octubre 09). Senkaku Islands dispute with China playing out as a PR battle. Japan Times. Consultado de http://www.japantimes.co.jp/text/nn20121009a6.html

- $\quad$ Fackler, M \& Johnson, I. (2012, Septiembre 20). Sleepy Islands and a Smoldering Dispute. The New York Times. Consultado de http://www.nytimes.com/2012/09/21/world/asia/japan-china-trade-tiescomplicate-island-dispute.html?pagewanted $=$ all $\&_{-} \mathrm{r}=0$

- Maya, K. (2010, Diciembre 08). Ishigaki fisherman fret over Senkaku encroachment. The Japan Times. Consultado de http://www.japantimes.co.jp/text/nn20101208f2.html

- $\quad$ Ogura, J, \& Mullen, J. (2012, Septiembre 19). Fresh anti-japanese protests in China on symbolic anniversary. CNN. Consultado de http://edition.cnn.com/2012/09/18/world/asia/china-japan-islandsdispute/index.html

- Tratado de Okinawa - 1971. (1971, Junio 17). Consultado 4 de Noviembre de 2012, de http://www. niraikanai.wwma.net/pages/archive/rev71.html

- $\quad$ Panetta tells China that Senkakus under Japan- U.S. Security Treaty. (2012, Septiembre 21). TheAsahiShimbun. Consultado de http://ajw.asahi.com/article/asia/china/AJ201209210061

- UnitedNations. (1951, Septiembre 08). Tratado de San Francisco. Consultado 13 de Octubre de 2012, de http://treaties.un.org/doc/Publication/UNTS/Nolume\%20136/volume-136-I-1832-English.pdf 